\title{
Outcomes Related to the Use of Frozen Plasma or Pooled Solvent/Detergent-Treated Plasma in Critically Ill Children
}

Camazine, Maraya N ; Karam, Oliver ; Colvin, Ryan ; Leteurtre, Stephane ; Demaret, Pierre ; Tucci, Marisa ; Muszynski, Jennifer A ; Stanworth, Simon ; Spinella, Philip C ; PlasmaTV investigators ; Pediatric Critical Care Blood Research Network

\begin{abstract}
OBJECTIVE: To determine if the use of fresh frozen plasma/frozen plasma 24 hours compared to solvent detergent plasma is associated with international normalized ratio reduction or ICU mortality in critically ill children. DESIGN: This is an a priori secondary analysis of a prospective, observational study. Study groups were defined as those transfused with either fresh frozen plasma/frozen plasma 24 hours or solvent detergent plasma. Outcomes were international normalized ratio reduction and ICU mortality. Multivariable logistic regression was used to determine independent associations. SETTING: One hundred one PICUs in 21 countries. PATIENTS: All critically ill children admitted to a participating unit were included if they received at least one plasma unit during six predefined 1-week (Monday to Friday) periods. All children were exclusively transfused with either fresh frozen plasma/frozen plasma 24 hours or solvent detergent plasma. INTERVENTIONS: None. MEASUREMENTS AND MAIN RESULTS: There were 443 patients enrolled in the study. Twenty-four patients (5\%) were excluded because no plasma type was recorded; the remaining 419 patients were analyzed. Fresh frozen plasma/frozen plasma 24 hours group included 357 patients, and the solvent detergent plasma group included 62 patients. The median (interquartile range) age and weight were 1 year (0.2-6.4) and $9.4 \mathrm{~kg}(4.0-21.1)$, respectively. There was no difference in reason for admission, severity of illness score, pretransfusion international normalized ratio, or lactate values; however, there was a difference in primary indication for plasma transfusion $(\mathrm{p}<0.001)$. There was no difference in median (interquartile range) international normalized ratio reduction, between fresh frozen plasma/frozen plasma 24 hours and solvent detergent plasma study groups, $-0.2(-0.4$ to 0$)$ and $-0.2(-0.3$ to 0$)$, respectively $(\mathrm{p}=0.80)$. ICU mortality was lower in the solvent detergent plasma versus fresh frozen plasma/frozen plasma 24 hours groups, $14.5 \%$ versus $29.1 \% \%$, respectively $(\mathrm{p}=0.02)$. Upon adjusted analysis, solvent detergent plasma transfusion was independently associated with reduced ICU mortality (odds ratio, 0.40; 95\% CI, 0.16-0.99; p = 0.05). CONCLUSIONS: Solvent detergent plasma use in critically ill children may be associated with improved survival. This hypothesis-generating data support a randomized controlled trial comparing solvent detergent plasma to fresh frozen plasma/frozen plasma 24 hours.
\end{abstract}

DOI: https://doi.org/10.1097/PCC.0000000000001149

Posted at the Zurich Open Repository and Archive, University of Zurich

ZORA URL: https://doi.org/10.5167/uzh-146928

Journal Article

Published Version

Originally published at: 
Camazine, Maraya N; Karam, Oliver; Colvin, Ryan; Leteurtre, Stephane; Demaret, Pierre; Tucci, Marisa; Muszynski, Jennifer A; Stanworth, Simon; Spinella, Philip C; PlasmaTV investigators; Pediatric Critical Care Blood Research Network (2017). Outcomes Related to the Use of Frozen Plasma or Pooled Solvent/Detergent-Treated Plasma in Critically Ill Children. Pediatric Critical Care Medicine, 18(5):e215e223.

DOI: https://doi.org/10.1097/PCC.0000000000001149 


\title{
Outcomes Related to the Use of Frozen Plasma or Pooled Solvent/Detergent-Treated Plasma in Critically III Children*
}

\author{
Maraya N. Camazine, BS ${ }^{1}$; Oliver Karam, MD, $\mathrm{PhD}^{2,3}$; Ryan Colvin, $\mathrm{MPH}^{1}$; \\ Stephane Leteurtre, $\mathrm{MD}, \mathrm{PhD}^{3,4}$; Pierre Demaret, $\mathrm{MD}, \mathrm{MSc}^{3,5}$; Marisa Tucci, $\mathrm{MD}^{6}$; \\ Jennifer A. Muszynski, MD; Simon Stanworth, MD $^{8,9}$; Philip C. Spinella, MD, FCCM ${ }^{1}$; on behalf of \\ the PlasmaTV Investigators and the Pediatric Critical Care Blood Research Network (Blood Net)
}

\begin{abstract}
Objective: To determine if the use of fresh frozen plasma/frozen plasma 24 hours compared to solvent detergent plasma is associated with international normalized ratio reduction or ICU mortality in critically ill children.

Design: This is an a priori secondary analysis of a prospective, observational study. Study groups were defined as those transfused with either fresh frozen plasma/frozen plasma 24 hours or solvent detergent plasma. Outcomes were international normalized ratio reduction and ICU mortality. Multivariable logistic regression was used to determine independent associations.
\end{abstract}

\section{*See also p. 496.}

${ }^{1}$ Division of Critical Care, Department of Pediatrics, Washington University in St. Louis, St. Louis, MO.

${ }^{2}$ Pediatric Intensive Care Unit, Geneva University Hospital, Geneva, Switzerland.

${ }^{3}$ University of Lille-Nord-de-France, EA 2694-Santé Publique: épidémiologie et qualité des soins, F-59000 Lille, France.

${ }^{4}$ Pediatric Intensive Care Unit, Centre Hospitalier Universitaire (CHU) Lille, F-59000 Lille, France.

${ }^{5}$ Division of Pediatric Critical Care Medicine, Department of Pediatrics, $\mathrm{CHC}$, Liège, Belgium.

${ }^{6}$ Division of Pediatric Critical Care Medicine, Department of Pediatrics, Sainte-Justine Hospital, University of Montreal, Montreal, QC, Canada.

${ }^{7}$ Division of Critical Care Medicine, Department of Pediatrics, Nationwide Children's Hospital, Columbus, $\mathrm{OH}$.

${ }^{8} \mathrm{NHS}$ Blood and Transplant/Oxford University Hospitals NHS Foundation Trust, John Radcliffe Hospital, Oxford, United Kingdom.

'University of Oxford, Oxford, United Kingdom.

PlasmaTV Investigators are listed in the Acknowledgments section.

Dr. Spinella received funding from consulting for Octapharma, Cerus, and TerumoBCT. The remaining authors have disclosed that they do not have any potential conflicts of interest.

Address requests for reprints to: Philip C. Spinella, MD, FCCM, Washington University School of Medicine at Washington University Medical Center, Northwest Tower Campus Box 8116, 660 South Euclid Avenue, St. Louis, MO, 63110. E-mail: Spinella_p@kids.wustl.edu

Copyright (C) 2017 by the Society of Critical Care Medicine and the World Federation of Pediatric Intensive and Critical Care Societies

DOI: 10.1097/PCC.0000000000001149
Setting: One hundred one PICUs in 21 countries.

Patients: All critically ill children admitted to a participating unit were included if they received at least one plasma unit during six predefined 1-week (Monday to Friday) periods. All children were exclusively transfused with either fresh frozen plasma/frozen plasma 24 hours or solvent detergent plasma.

Interventions: None.

Measurements and Main Results: There were 443 patients enrolled in the study. Twenty-four patients (5\%) were excluded because no plasma type was recorded; the remaining 419 patients were analyzed. Fresh frozen plasma/frozen plasma 24 hours group included 357 patients, and the solvent detergent plasma group included 62 patients. The median (interquartile range) age and weight were 1 year $(0.2-6.4)$ and $9.4 \mathrm{~kg}$ (4.0-21.1), respectively. There was no difference in reason for admission, severity of illness score, pretransfusion international normalized ratio, or lactate values; however, there was a difference in primary indication for plasma transfusion $(p<0.001)$. There was no difference in median (interquartile range) international normalized ratio reduction, between fresh frozen plasma/frozen plasma 24 hours and solvent detergent plasma study groups, $-0.2(-0.4$ to 0$)$ and -0.2 ( -0.3 to 0$)$, respectively $(p=0.80)$. ICU mortality was lower in the solvent detergent plasma versus fresh frozen plasma/frozen plasma 24 hours groups, $14.5 \%$ versus $29.1 \% \%$, respectively $(p=0.02)$. Upon adjusted analysis, solvent detergent plasma transfusion was independently associated with reduced ICU mortality (odds ratio, $0.40 ; 95 \% \mathrm{Cl}, 0.16-0.99 ; p=0.05)$.

Conclusions: Solvent detergent plasma use in critically ill children may be associated with improved survival. This hypothesis-generating data support a randomized controlled trial comparing solvent detergent plasma to fresh frozen plasma/frozen plasma 24 hours. (Pediatr Crit Care Med 2017; 18:e215-e223)

Key Words: children; critical illness; epidemiology; plasma transfusion; solvent detergent plasma 
$\mathrm{P}$ lasma products are transfused frequently. In 2013, $1,797,000$ plasma units were transfused in the United States in adults and children by American Association of Blood Banks (AABB) member hospitals (1). According to U.S. pediatric health information administrative databases, nearly $3 \%$ of all recorded pediatric admissions receive a plasma transfusion during their hospital stay (2). Unfortunately, plasma products are also often transfused unnecessarily in both children and adults $(3,4)$. This is important since there are risks associated with plasma use primarily in nonbleeding populations (5). Conversely, there is recent evidence indicating early plasma transfusion reduces death from hemorrhage in adult trauma patients (6). Therefore, the risk-to-benefit ratio of plasma transfusion is dependent upon the patient illness. However, it is also possible that the risk-to-benefit ratio of plasma transfusion depends upon the type of plasma transfused, since the characteristics of plasma vary widely according to processing methods (7).

There are multiple collection/processing methods for plasma products. These include, but are not limited to, fresh frozen plasma (FFP), frozen plasma 24 hours (FP24), and pooled solvent/detergent-treated plasma (SDP). FFP is defined as plasma separated and frozen from single donor within 8 hours of collection, and FP24 is defined as plasma collected, separated, and frozen within 24 hours of collection. SDP is a pooled plasma product developed to improve the safety profile of plasma. SDP is collected from over 1,000 donors who have been screened for nonenveloped viruses. The plasma product is filtered to remove cells, cell fragments, and aggregates, thus

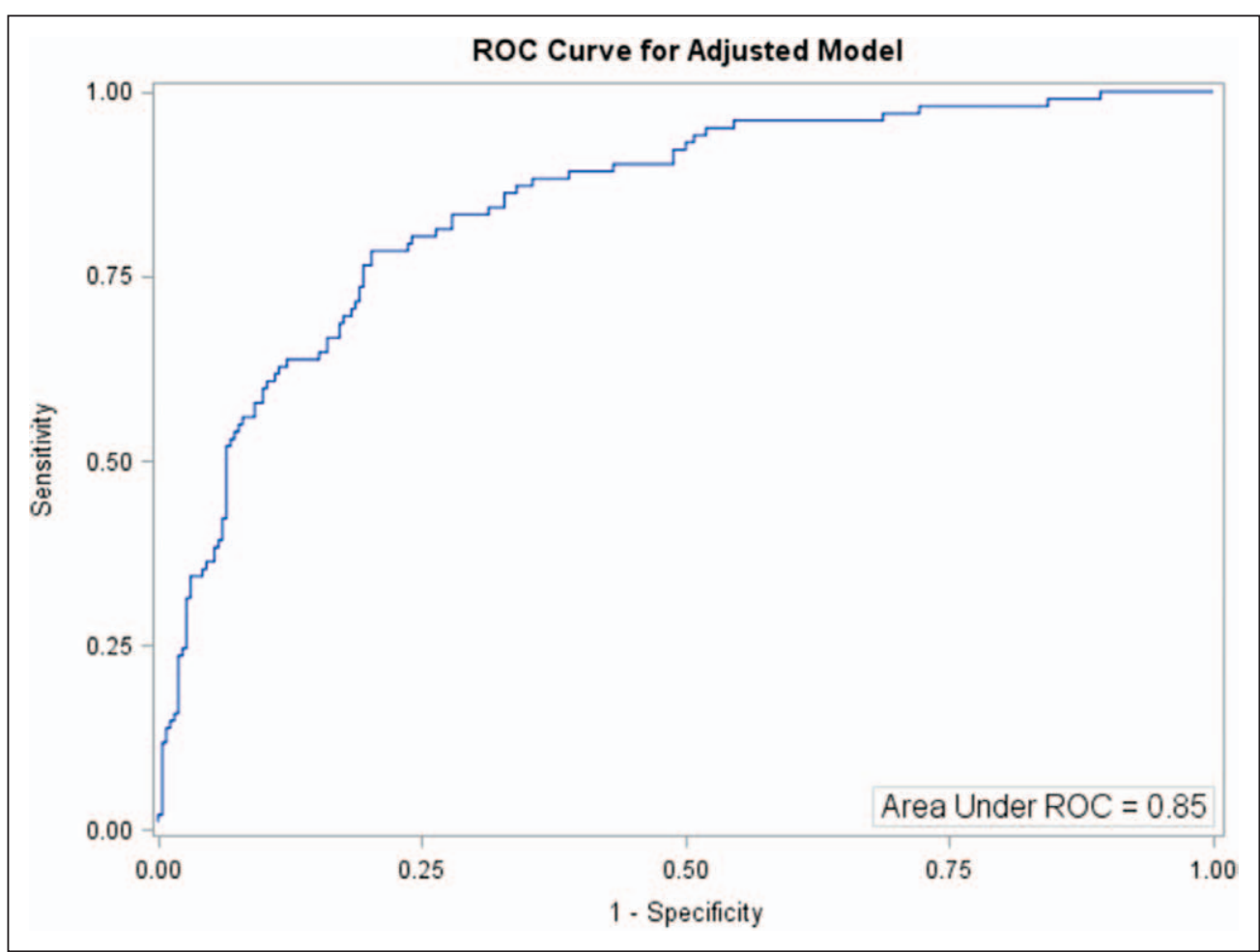

Figure 1. Receiver operating characteristic $(\mathrm{ROC})$ curve of the final multivariable model. The area under the curve was 0.85 (95\% Cl, 0.80-0.89). Model: Reason for PICU admission, day 1 Pediatric Logistic Organ Dysfunction-2 score, highest log lactate on day of transfusion, plasma type transfused, continuous renal replacement therapy during transfusion, log total volume of plasma per kilogram. removing intracellular pathogens. The product is also treated with tri-N-butyl-phosphate for pathogen and WBC inactivation. Additionally, the product undergoes a second round of sterile filtration, which removes bacteria and parasites. The plasma product is then packed and frozen in standardized units. There are no large, multicenter, high-quality randomized controlled trials (RCTs) comparing efficacy or safety of FFP/FP24 to SDP products, especially in children. FFP and FP24 are most frequently administered in the United States, whereas in Europe, SDP is more commonly transfused. Pooled SDP has been licensed in Europe for decades and more recently licensed in the United States in 2013.

With the frequent use of plasma in children, it is important to determine if different plasma processing methods can improve efficacy and safety. Our primary objectives for this hypothesis-generating study were to determine 1) if plasma processing relates to efficacy, by evaluating change in international normalized ratio (INR) post plasma transfusion and 2) if plasma processing is associated with ICU mortality.

\section{METHODS}

This study is approved by the local Institutional Review Board at Washington University School of Medicine. This is an a priori planned secondary analysis of a prospective, observational study aimed to determine if there was an independent association between the type of plasma transfused and INR reduction or ICU mortality in critically ill children. Data were extracted from the recently published PlasmaTV cohort (3). One hundred one PICUs in 21 countries participated in the study. Study data were collected from six predefined 1-week (Monday to Friday) periods. All critically ill children admitted to a participating ICU were included if they received at least one plasma unit during any of these six 1-week periods. Plasma transfused outside of the ICU was not captured in this study. In total, 13,192 children presented to site PICUs during the predefined weeks; of these children, 443 children received at least one plasma transfusion and enrolled in the study (3). Twenty-four patients (5\%) were excluded from our analysis because no plasma type was recorded; the remaining 419 patients were analyzed. The PlasmaTV study collected laboratory data that were ordered clinically. It did not require pre and post plasma transfusion laboratory analyses to be performed. 
Clinical indications for plasma transfusion were categorized as follows: 1) Critical bleeding: massive bleeding (transfusion of all blood products $>80 \mathrm{~mL} / \mathrm{kg}$ within $24 \mathrm{hr}$ ), bleeding in specific sites (intracranial, intraocular, retroperitoneal, intraspinal, pericardial, and nontraumatic intra-articular), or bleeding requiring a surgical intervention or drainage (e.g., hemothorax requiring drainage). 2) Minor bleeding: minor surgical bleeding (wound, drain, etc.) or minor nonsurgical bleeding (endotracheal tube secretions, nasogastric tube, urine, etc.). 3) Planned surgery or procedures: central venous catheter, pleural drain, etc. 4) High risk of postoperative bleeding: as defined by the intensivist. 5) No bleeding, no planned procedure: hypovolemia, abnormal coagulation tests, factor or component replacement, at high risk of bleeding due to nonsurgical reasons.
Descriptive statistics were used to report continuous and dichotomous variables. Continuous variables are reported as median and interquartile range (IQR). Dichotomous variables are reported as $n(\%)$. Unadjusted comparisons of continuous and categorical variables used Wilcoxon rank sum test and Fisher exact test, respectively. To assess which variables were potential confounders for ICU mortality, variables were compared for patients who survived or did not survive in the ICU.

To establish independent associations between variables recorded and mortality, a multivariable logistic regression model was developed. The adjusted model was determined by forward and backward stepwise selection of baseline characteristics associated with mortality. Candidate variables must have had a $p$ value of less than or equal to 0.20 on unadjusted analyses, which

\section{TABLE 1. Relationship Between Type of Plasma and Population Characteristics}

\begin{tabular}{|c|c|c|c|}
\hline Candidate Independent Variables & $\begin{array}{l}\text { Fresh Frozen Plasma/ } \\
\text { Frozen Plasma } 24 \mathrm{hr} \\
\qquad(n=357)\end{array}$ & $\begin{array}{l}\text { Pooled Solvent/Deter- } \\
\text { gent-Treated Plasma } \\
\qquad(n=62)\end{array}$ & p \\
\hline Gender, $n(\%)$ & & & 0.78 \\
\hline Male & $152(42.6)$ & $25(40.3)$ & \\
\hline Female & $205(57.4)$ & $37(59.7)$ & \\
\hline Age, yr, median (IQR) & $1.1(0.2-6.4)$ & $0.8(0.3-6.3)$ & 0.80 \\
\hline Reason for admission, $n$ (\%) & & & 0.88 \\
\hline Medical only & $142(39.8)$ & $23(37.1)$ & \\
\hline Surgical only & $133(37.3)$ & $25(40.3)$ & \\
\hline Combination of medical and surgical & $82(23.0)$ & $14(22.6)$ & \\
\hline Primary indication for plasma transfusion, $n(\%)$ & & & $<0.001$ \\
\hline No bleeding, no procedure & $128(35.9)$ & $17(27.4)$ & \\
\hline Critical bleeding & $81(22.7)$ & $10(16.1)$ & \\
\hline Minor bleeding & $64(17.9)$ & $27(43.6)$ & \\
\hline Preparation/planned procedure & $44(12.3)$ & $6(10.0)$ & \\
\hline High risk of postoperative bleeding & $40(11.2)$ & $2(3.2)$ & \\
\hline $\begin{array}{l}\text { Pediatric Logistic Organ Dysfunction }-2 \text { score at transfusion, median } \\
\text { (IOR) }\end{array}$ & $7(5-10)$ & $7(5-9)$ & 0.26 \\
\hline INR prior to transfusion, median (IOR) ${ }^{\mathrm{a}}$ & $1.6(1.3-2.0)$ & $1.5(1.2-1.9)$ & 0.89 \\
\hline Change in INR, posttransfusion minus pretransfusion, median (IQR) & $-0.2(-0.4$ to 0$)$ & $-0.2(-0.3$ to 0$)$ & 0.80 \\
\hline Highest lactate on day of transfusion, median $(I Q R)^{c}$ & $2.6(1.5-5.0)$ & $2.3(1.6-4.3)$ & 0.72 \\
\hline Extracorporeal life support during transfusion, $n$ (\%) & $36(10.1)$ & $4(6.5)$ & 0.49 \\
\hline Continuous renal replacement therapy during transfusion, $n(\%)$ & $28(7.8)$ & $6(9.7)$ & 0.62 \\
\hline Intermittent hemodialysis & $4(1.1)$ & $1(1.6)$ & 0.55 \\
\hline Total volume of plasma $(\mathrm{mL} / \mathrm{kg})$, median $(I \mathrm{OR})^{\mathrm{d}}$ & $21.4(11.1-55.4)$ & $15.0(10.0-40.9)$ & 0.07 \\
\hline Mortality, $n(\%)$ & $104(29.1)$ & $9(14.5)$ & 0.02 \\
\hline
\end{tabular}

INR = international normalized ratio, IQR = interquartile range.

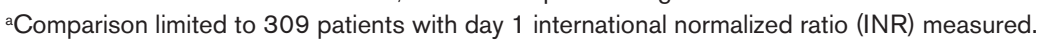

${ }^{b}$ Comparison limited to 270 patients with pre and posttransfusion INR measured.

${ }^{\mathrm{C}}$ Comparison limited to 368 patients with lactate on day of transfusion measured.

${ }^{\mathrm{d}}$ Comparison limited to 415 patients with total volume of plasma recorded. 
TABLE 2. Relationship Between ICU Mortality and Population Characteristics

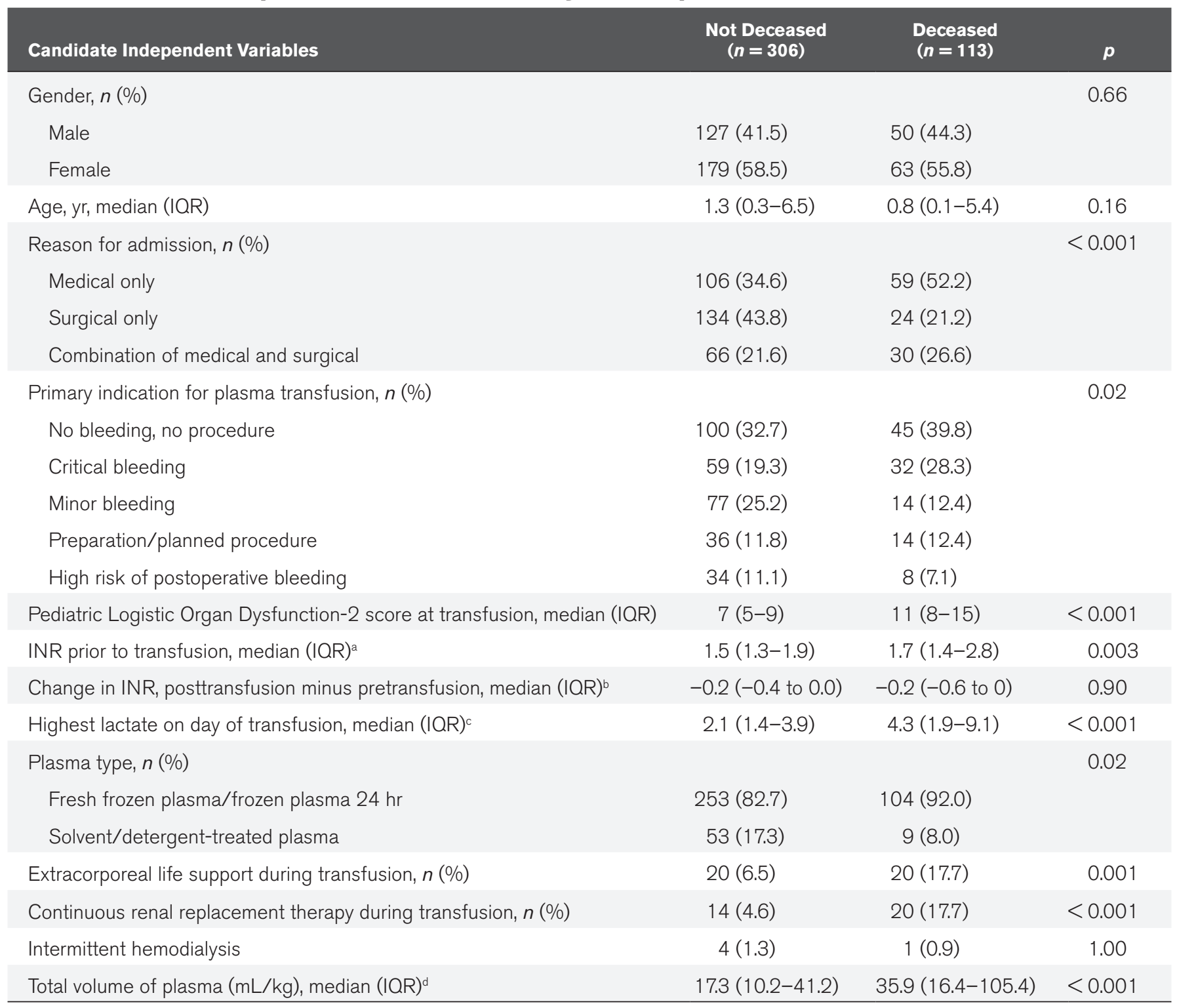

INR = international normalized ratio, IQR = interquartile range.

${ }^{a}$ Comparison limited to 309 patients with day 1 international normalized ratio (INR) measured.

${ }^{b}$ Comparison limited to 270 patients with pre and posttransfusion INR measured.

${ }^{\circ}$ Comparison limited to 368 patients with lactate on day of transfusion measured.

${ }^{\mathrm{d}}$ Comparison limited to 415 patients with total volume of plasma recorded.

included patient age, reason for PICU admission, indication for plasma transfusion, day 1 Pediatric Logistic Organ Dysfunction (PELOD)-2 score, extracorporeal life support (ECLS) and continuous renal replacement therapy (CRRT) during transfusion, lactate level, and total volume per kilogram of plasma during PICU stay. Lactate concentrations and total volume per kilogram of plasma transfused had nonnormal distributions and were hence log-transformed for use in regression analyses. INR prior to transfusion was not included in the multivariable model as this measure was not available for approximately $26 \%$ of the study population. These variables were then assessed with forward and backward stepwise models, with study group forced into the models. Inclusion threshold for the stepwise processes was set to $p$ value of less than 0.05 . Candidate variables that met the stepwise process threshold of $p$ value less than 0.05 in either stepwise model developed were included the final model. Area under the receiver operator characteristic (ROC) curve for the adjusted model was calculated to assess the model's predictive ability (Fig. 1). The Hosmer-Lemeshow goodness-of-fit test was also performed to assess whether or not the observed event rates match expected event rates in subgroups of the model population. Multicollinearity between variables was assessed and determined not to be of issue in this analysis. For comparison, unadjusted logistic regression models were generated for each candidate variable. All analyses were conducted using SAS 9.4 (SAS Institute, Cary, NC). 


\section{RESULTS}

There were 443 critically ill children enrolled in the study. Twentyfour patients $(5 \%)$ were excluded because no plasma type was recorded, and one patient $(0.2 \%)$ was transfused with Mirasoltreated plasma (TerumoBCT, Lakewood, CO); the remaining 419 patients were analyzed. Forty-two percent (177/419) of the patient population were males. The median (IQR) age and weight were 1.0 year $(0.2-6.4)$ and $9.4 \mathrm{~kg}(4.0-21.1)$, respectively. Mortality rate in all patients was $27 \%(113 / 419)$. Thirty-nine percent $(165 / 419)$ of patients were admitted for medical reasons only, 38\% (158/419) were admitted for surgical reasons only, and 23\% (96/419) were admitted for a combination of medical and surgical reasons. All children received either FFP/FP24 $(n=357)$ or SDP $(n=62)$ exclusively. No patients received a mixture of FFP/FP24 and SDP.

When comparing the two study groups, there was no difference in gender, age, or reason for admission. There was a significant difference between the two groups for the primary indication for plasma transfusion $(p \leq 0.001)$. The FFP/FP24 group had a higher proportion of patients were transfused for critical bleeding, no bleeding, or planned procedure, compared to the SDP group. The SDP group had a higher proportion of patients transfused for minor bleeding compared to the FFP/ FP24 group (Table 1). More patients were transfused SDP for any type of bleeding (critical and minor) than were transfused FFP/FP24, 59.7\% versus 40.6\%, respectively ( $p=0.009$ ). There was no difference in illness severity parameters between the two groups such as PELOD-2 scores, pretransfusion INR, or highest lactate on day of transfusion (Table 1). The difference in total volume of plasma transfused in the FFP/FP24 group compared to the SDP group approached significance $(p=0.07)$. Although there was no difference in INR reduction between both study groups, there was reduced mortality in the SDP group compared to the FFP/FP24 group, 14.5\% (9/62) versus $29.1 \%(104 / 357)$, respectively $(p=0.02)$ (Table 1$)$.

Variables associated with ICU mortality were reason for admission, primary indication for plasma transfusion, PELOD-2 score at transfusion, INR prior to transfusion, highest lactate on day of transfusion, type of plasma type transfused, total volume of plasma $(\mathrm{mL} / \mathrm{kg})$ transfused, ECLS use, and CRRT use (Table 2).

When comparing the two study groups, we also analyzed ICU mortality for each transfusion indication (Table 3). For each plasma transfusion indication evaluated, the ICU mortality rate was lower in the SDP group, though these values did not reach statistical significance. There was no one plasma indication that was significant for a reduction in ICU mortality with SDP, although there was a numerical reduction in ICU mortality in each group when SDP was transfused.

Table 4 indicates the variables associated with ICU mortality by univariate regression analysis. In this unadjusted model, use of SDP was associated with reduced ICU mortality (odds ratio [OR], $0.41 ; 95 \% \mathrm{CI}, 0.20-0.87 ; p=0.02$ ).

Multivariable logistic regression showed a two-fold decrease in mortality after adjustment for confounding variables that were marginally significant (OR, 0.40; 95\% CI, 0.16-0.99; $p=0.05$ ) (Table 5). As expected, severity of illness as measured by the PELOD-2 score or need for CRRT were both independently associated with increased ICU mortality. The volume per kilogram of either plasma product was also independently associated with increased ICU mortality. Due to nonsignificance during the stepwise process, upon adjusted analysis, plasma transfusion indication was removed from the model. The Hosmer-Lemeshow goodness-of-fit test $(p=0.40)$ demonstrates that the data fit the logistic distribution. The adjusted model shows good predictive ability, with an area under the ROC equals to 0.85 .

\section{DISCUSSION}

This prospective, observational study is the first analysis examining the association between plasma processing methods and outcomes in critically ill children. Our analysis indicates that although no difference in INR reduction was observed, upon adjustment, SDP transfusion was independently associated with a two-fold reduction in ICU mortality when compared with FFP/FP24 products.

The literature regarding the effect of plasma on outcomes in pediatric patients is scant. One pediatric study examining FFP and FP24 use reported a $41 \%$ prevalence of new or progressive multiple organ dysfunction syndrome in patients transfused

\section{TABLE 3. ICU Mortality Per Indication of Plasma Transfusion by Study Group}

\begin{tabular}{|c|c|c|}
\hline Indication & $\begin{array}{c}\text { ICU Mortality, } \\
n(\%)\end{array}$ & $p$ \\
\hline No bleeding, no procedure & & 0.58 \\
\hline FFP/FP24 $(n=128)$ & $41(32.0)$ & \\
\hline $\operatorname{SDP}(n=17)$ & $4(23.5)$ & \\
\hline Critical bleeding & & 0.48 \\
\hline FFP/FP24 $(n=81)$ & $30(37.0)$ & \\
\hline $\operatorname{SDP}(n=10)$ & $2(20.0)$ & \\
\hline Minor bleeding & & 0.22 \\
\hline FFP/FP24 $(n=64)$ & $12(18.8)$ & \\
\hline $\operatorname{SDP}(n=27)$ & $2(7.4)$ & \\
\hline Preparation/planned procedure & & 0.66 \\
\hline FFP/FP24 $(n=44)$ & $13(30.0)$ & \\
\hline $\operatorname{SDP}(n=6)$ & $1(16.7)$ & \\
\hline High risk of postoperative bleeding & & 1.00 \\
\hline FFP/FP24 $(n=40)$ & $8(20.0)$ & \\
\hline $\operatorname{SDP}(n=2)$ & $0(0.0)$ & \\
\hline All indications & & 0.02 \\
\hline FFP/FP24 $(n=357)$ & $104(29.1)$ & \\
\hline $\operatorname{SDP}(n=62)$ & $9(14.5)$ & \\
\hline
\end{tabular}

FFP/FP24 = fresh frozen plasma/frozen plasma $24 \mathrm{hr}, \mathrm{SDP}=$ solvent/ detergent-treated plasma. 


\section{TABLE 4. Unadjusted Logistic Regression Models of Independent Variables and ICU Mortality}

Candidate Independent Variables

Reason for admission

Surgical only

Medical only

Combination of medical and surgical

Primary indication for plasma transfusion

No bleeding, no procedure

Critical bleeding

Minor bleeding

Preparation/planned procedure

High risk of postoperative bleeding

Pediatric Logistic Organ Dysfunction-2 score at transfusion

International normalized ratio prior to transfusion ${ }^{a}$

Highest log lactate on day of transfusion ${ }^{b}$

Plasma type

Fresh frozen plasma/frozen plasma $24 \mathrm{hr}$

Solvent/detergent-treated plasma

Extracorporeal life support during transfusion, yes vs no

Continuous renal replacement therapy during transfusion, yes vs no

Log total volume of plasma $(\mathrm{mL} / \mathrm{kg})^{c}$
OR $(95 \% \mathrm{Cl})$

p

0.0002

Reference

$3.11(1.81-5.33)$

$<0.0001$

$2.54(1.38-4.68)$

0.003

0.02

Reference

$1.21(0.69-2.10)$

0.51

$0.40(0.21-0.79)$

0.008

$0.86(0.43-1.76)$

0.69

$0.52(0.22-1.22)$

0.13

$1.31(1.22-1.40)$

$<0.0001$

1.39 (1.15-1.68)

0.0006

$2.27(1.71-3.01)$

$<0.0001$

$\mathrm{OR}=$ odds ratio.

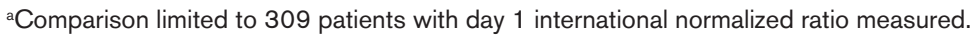

${ }^{b}$ Comparison limited to 368 patients with lactate on day of transfusion measured.

${ }^{\mathrm{c}}$ Comparison limited to 415 patients with total volume of plasma recorded.

with FFP/FP24 compared to an $8 \%$ incidence for those not transfused at all $(p<0.001)$. There was a $17 \%$ incidence of nosocomial infections in the FFP/FP24 group compared to $3.7 \%$ incidence in patients not transfused $(p=0.04)$. There was no significant difference in 28-day mortality; however, there was a significant difference in the length of ICU stay (8).

In adults, plasma transfusion with FFP/FP24 in predominantly nonbleeding or nonmassive bleeding cohorts has independently been associated with increased morbidity (5) and mortality in trauma patients $(9,10)$, patients with gastrointestinal bleeding (11), and critically ill patients (12). FFP/FP24 transfusion has also been associated with increased incidence of acute respiratory distress syndrome (13), transfusion-related acute lung injury (TRALI) (14), transfusion-associated circulatory overload (15), nosocomial infections (16), and multiple organ failure in nonmassively bleeding patients (17). Conversely, in a secondary analysis of a large RCT, increased plasma use relative to RBCs with FFP/FP24 in massively bleeding adult trauma patients reduces death from hemorrhage and the time to hemostasis $(6,18)$.

Although similar types of analyses have not been performed with SDP, European data in general indicate that it has an increased safety profile compared to FFP/FP24 products.
In one observational study, patients who were transfused a pooled SDP product had an $83 \%$ reduction in adverse reactions and no cases of TRALI compared to those receiving FFP (19). Another analysis evaluating the risk associated with FFP and Octaplas (Octapharma, Lachen, Switzerland), a SDP product, showed that in countries where Octaplas is transfused, there were no reported cases of TRALI compared to 22 cases reported where FFP is transfused (20). A recent small RCT compared SDP and FFP in orthotopic liver transplant patients in whom thromboelastography was used to direct the indication for plasma transfusion. In this study, there was a statistically significant reduction in the amount of plasma needed to correct coagulopathy in patients receiving Octaplas compared to those receiving FFP (21). Other data indicate that in adult patients undergoing open-heart surgery, SDP is equally as effective at correcting coagulopathy when compared with FFP (18).

Our results indicate no difference in efficacy between study groups for INR reduction. Possible reasons for this could be that INR reduction itself may not be the most accurate measure reflecting coagulation or there could simply be no difference in efficacy between the groups. Future studies comparing SDP 


\section{TABLE 5. Multiple Logistic Regression Model of Independent Variables and ICU Mortality}

Candidate Independent Variables

Reason for admission

Surgical only

Medical only

Combination of medical and surgical

Day 1 Pediatric Logistic Organ Dysfunction-2 score

Highest log lactate on day of transfusion

Plasma type

Fresh frozen plasma/frozen plasma $24 \mathrm{hr}$

Solvent/detergent-treated plasma

Continuous renal replacement therapy during transfusion, yes vs no

Log total volume of plasma $(\mathrm{mL} / \mathrm{kg})$

OR = odds ratio.

Variables placed in the model that were not reported due to nonsignificance include indication for plasma transfusion, patient age, and use of extracorporeal life support.

and FFP/FP24 products should incorporate other measures that reflect thrombin formation such as viscoelastic measures and thrombin generation assays, in addition to reducing clinically significant bleeding and associated clinical outcomes, to further evaluate plasma efficacy. Thrombin generation potential between samples from FFP and SDP units has been demonstrated to be different in "in vitro" experiments, especially when FFP remained thawed for up to 5 days (7). In this report, total thrombin generation of thawed plasma is reduced at day 5 compared to day 0 , and there were more platelet and RBC microparticles in FFP compared to SDP. Since our analysis and previously published studies that evaluated the association between FFP/FP24 and outcomes have not reported the storage duration of "thawed plasma," it is not possible to assess if this has contributed to the results reported. It is important for future studies to record the storage duration of thawed plasma since a recent $\mathrm{AABB}$ survey indicates that approximately $30-53 \%$ of all plasma transfused is thawed and then stored between 1 and 5 days before transfusion in the United States $(1,22)$. One hypothesis is that the reduced thrombin generation and increased platelet and RBC-derived microparticles affect the efficacy and safety of FFP/FP24 products, especially when thawed and stored, compared to SDP (7). This hypothesis requires analysis in prospective human studies.

Although there was no difference in INR reduction, our results suggest there may be an association with reduced ICU mortality with SDP compared to the FFP/FP24 group. The reduction in ICU mortality, according to the type of plasma transfused, was diffusely distributed among the indications for plasma transfusion, suggesting that the association was not limited to a specific indication (Table 3). The lack of statistical significance in this subgroup analysis may be due to the reduced power with a smaller sample size in each subgroup. The potential reduction in ICU mortality could again be due to the improved safety profile of SDP, specifically the

$$
\text { Reference }
$$

2.76 (1.39-5.46)

0.004

2.09 (0.94-4.65)

$1.24(1.14-1.35)$

$<0.0001$

$1.45(1.00-2.10)$

0.05

0.05

\section{Reference}

$0.40(0.16-0.99)$

0.05

2.50 (1.08-5.79)

0.03

1.59 (1.26-2.00)

$<0.0001$ pooling process, which dilutes anti-human leukocyte antigen and anti-human neutrophil antigen antibodies, and the extraction phase and filtering, which removes microparticles and biologically reactive mediators. The potential mechanisms are speculative and require investigation. In order to further evaluate the potential effects on efficacy and safety of SDP versus FFP/FP24, RCTs are needed that include mechanistic ancillary studies. We have estimated the sample size needed to assess the relationship between plasma type and mortality in a prospective study. Based on an OR of 0.4 and ICU mortality of 0.29 (95\% CI, 0.24-0.35), we estimate a sample size of 226 to 310 participants would be required.

The independent association of increased volume per kilogram of either FFP/FP24 or SDP transfused with poor outcomes in our analysis is consistent with previous reports with FFP in pediatric patients (8). It is unknown if there is a doseeffect of plasma on worse outcomes in certain clinical scenarios or if these findings represent confounding by indication.

Limitations of this prospective study include its lack of randomization and risk of selection bias. The differences in the indication for plasma between study groups could be a source of bias, but plasma indication was removed from the model for ICU mortality due to nonsignificance, and critical bleeding, which was more common as an indication for transfusion in the FFP/FP2 group, was not associated with ICU mortality on univariate analysis (Table 4). The inability to adjust for RBC or platelet volumes transfused, as well as for center effect, is also limitations. The lack of cause of death for patients in the analysis also reduced our ability to interpret our results. The noninclusion of plasma transfused outside the ICU, for example, during cardiopulmonary bypass, is another limitation. The low number of patients who received SDP may have also reduced the power of our analysis as well as its generalizability. In addition, we cannot exclude that patients received a different type of plasma before the study period started; therefore, although the plasma types were exclusive to one group 
or another during the study, we cannot confirm it was prior to the initiation of data collection for the PlasmaTV study. However, this was an a priori planned prospective study, from 21 countries and with large patient cohort with a strong multivariable logistic regression model that is hypothesis generating in nature.

\section{CONCLUSIONS}

In this large, international, prospective cohort study of critically ill children, SDP had a similar effect on INR reduction when compared with FFP/FP24 products and was marginally significant for a reduction in ICU mortality. Our results support the development of prospective trials to further analyze the efficacy and safety of SDP transfusions compared to FFP or FP24 in critically ill children.

\section{ACKNOWLEDGMENTS}

We thank all of the PlasmaTV Investigators. PlasmaTV Investigators: Australia: Warwick Butt, Carmel Delzoppo, Kym Bain (Royal Childrens Hospital, Melbourne, VIC); Simon Erickson, Nathan Smalley (Princess Margaret Hospital for Children, Perth, WA); Tavey Dorofaeff, Debbie Long (Royal Childrens Hospital, Brisbane, QLD); and Nathan Smalley, Greg Wiseman (Townsville Hospital, Townsville, QLD). Belgium: Stéphan Clément de Cléty, Caroline Berghe (Cliniques Universitaires SaintLuc, Brussels); Annick de Jaeger (Princess Elisabeth Children's University Hospital, Ghent); Pierre Demaret, Marc Trippaerts (CHC-CHR, Liège); Ariane Willems, ShancyRooze (Hôpital Universitaire des enfants Reine Fabiola, Brussels); and Jozef De Dooy (Antwerp University Hospital, Edegem). Canada: Elaine Gilfoyle, Lynette Wohlgemuth (Alberta Children's Hospital, Calgary, AB); Marisa Tucci, Mariana Dumitrascu (CHU Sainte-

Justine, Montréal, QC); Davinia Withington, Julia Hickey (Montreal Children's Hospital, Montreal, QC); Karen Choong, Lois Sanders (McMaster Children's Hospital, Hamilton, ON); Gavin Morrison (IWK Health Centre, Halifax, NS); Janice Tijssen (Children's Hospital, London Health Sciences Centre, London, ON); David Wensley, Gordon Krahn (British Columbia Children's Hospital, Vancouver, BC); Marc-Andre Dugas, Louise Gosselin (Centre Mère Enfant Soleil, CHU de Québec, Québec, QC); and Miriam Santschi (CHUS, Sherbrooke, QC). Chile: Bettina Von Dessauer, Nadia Ordenes (Hospital De Niños Roberto Del Río, Santiago). Denmark: Arash Afshari, Lasse Hoegh Andersen, Jens Christian Nilsson, Mathias Johansen, Anne-Mette Baek Jensen (Rigshospitalet, University of Copenhagen, Copenhagen). Ecuador: Santiago Campos Mino, Michelle F. Grunauer (Hospital de los Valles, Universidad San Francisco de Quito, Quito). France: Nicolas Joram (Hôpital mère enfant, Nantes); Nicolas Roullet-Renoleau (Hôpital Gatien de Clocheville, CHU Tours, Tours); Etienne Javouhey, Fleur Cour-Andlauer, Aurélie Portefaix (Hôpital Femme Mère Enfant, Hospices Civils de Lyon, Lyon); Olivier Brissaud, Julie Guichoux (Hôpital des Enfants, Bordeaux); Valérie Payen
(CHU Grenoble, Grenoble);Pierre-Louis Léger (Hôpital Armand-Trousseau, Paris); Mickael Afanetti (Hopitaux Pédiatriques CHU Lenval, Nice); Guillaume Mortamet (Hôpital Necker, Paris); Matthieu Maria (Hôpital d'Enfants CHRU de Nancy, Nancy); Audrey Breining (Hopitaux Universitaires de Strasbourg, Strasbourg); Pierre Tissieres (Hôpital KremlinBicêtre, Paris); Aimée Dorkenoo (CHRU Lille, Lille); and Anna Deho (Hôpital Robert Debré, Paris). Germany: Harry Steinherr (Medizinische Hochschule Hannover, Hannover). Greece: Filippia Nikolaou (Athens Children Hospital P\&A Kyriakou, Athens). Italy: Anna Camporesi (Children Hospital VittoreBuzzi, Milano) and Federica Mario (Department of Women's and Children's Health, Padua). Japan: Tatsuya Kawasaki, Shinya Miura (Shizuoka Children's Hospital, Shizuoka City). New Zealand: John Beca, Miriam Rea, Claire Sherring, Tracey Bushell (Starship Children's Hospital, Auckland). Norway: Gunnar Bentsen (Oslo University Hospital-Rikshospitalet, Oslo). Portugal: Alexandra Dinis (Hospital Pediátrico-CHUC, Coimbra); Gabriela Pereira (UCIP Hospital Dona Estefânia, Lisbon); Marisa Vieira (Hospital de Santa Maria, Lisbon); and Marta Moniz (Hospital Prof. Dr Fernando Fonseca, Amadora). Saudi Arabia: Saleh Alshehri (King Saud Medical City, Riyadh) and Manal Alasnag, Ahmad Rajab (King Fahd Armed Forces Hospital, Jeddah). Slovakia: Maria Pisarcikova (DFN Kosice, Kosice). Spain: Iolanda Jordan (Hospital Sant Joan de Déu, Barcelona); Joan Balcells (Hospital Valld'Hebron, Barcelona); Antonio Perez-Ferrer, Jesús de Vicente Sánchez, Marta Vazquez Moyano (La Paz University Hospital, Madrid); Antonio Morales Martinez (Malaga Regional University Hospital, Malaga); Jesus Lopez-Herce, Maria Jose Solana (Hospital General Universitario Gregorio Marañón, Madrid); Jose Carlos Flores González (Puerta del Mar University Hospital, Cadiz); Maria Teresa Alonso (Hospital Virgen del Rocío, Sevilla); and Manuel Nieto Faza (Hospital Universitario Cruces, Bilbao). Switzerland: Marie-Hélène Perez, Vivianne Amiet (CHUV, Lausanne); Carsten Doell (Kinderspital Zürich, Zürich); and Alice Bordessoule (Geneva University Hospital, Geneva). The Netherlands: Suzan Cochius-den Otter, Berber Kapitein (Erasmus MCSophia Children's Hospital, Rotterdam) and Martin Kneyber (Beatrix Children's Hospital, Groningen). United Kingdom: Joe Brierley, Vanessa Rea, Stephen McKeever (Great Ormond Street, London); Andrea Kelleher (Royal Brompton Hospital, London); Barney Scholefield, Anke Top, Nicola Kelly, Satnam Virdee (Birmingham Children's Hospital, Birmingham); Peter Davis, Susan George (Bristol Royal Hospital for Children, Bristol); Kay C. Hawkins, Katie McCall, Victoria Brown (Royal Manchester Children's Hospital, Manchester); Kim Sykes (University Hospital Southampton, Southampton); Richard Levin, Isobel MacLeod (Yorkhill Childrens Hospital, Glasgow); Marie Horan, Petr Jirasek (Alder Hey Children's Hospital, Liverpool); David Inwald, Amina Abdulla, Sophie Raghunanan (Imperial College Healthcare, London); Bob Taylor (Royal Belfast Hospital for Sick Children, Belfast); and Alison Shefler, Hannah Sparkes 
(Oxford University Hospitals, Oxford). United States: Sheila Hanson, Katherine Woods, David Triscari, Kathy Murkowski (Children's Hospital of Wisconsin, Milwaukee, WI); Caroline Ozment (Duke University, Durham, NC); Marie Steiner, Dan Nerheim, Amanda Galster (University of Minneapolis, Minneapolis, MN); Renee Higgerson, LeeAnn Christie (Dell Children's Medical Center, Austin, TX); Phil Spinella, Daniel Martin, Liz Rourke (Washington University in St. Louis, Saint Louis, MO); Jennifer Muszynski, Lisa Steele (Nationwide Children's Hospital, Columbus, OH); Samuel Ajizian, Michael Conor McCrory (Wake Forest School of Medicine, Winston-Salem, NC); Kevin O'Brien, Christopher Babbitt, Erin Felkel, Glenn Levine (Miller Children's Hospital Long Beach, Long Beach, CA); Edward J. Truemper, Machelle Zink (Children's Hospital and Medical Center, Omaha, NE); Marianne Nellis (NYPHWeill Cornell Medical College, New York, NY); Neal J. Thomas, Debbie Spear (Penn State Hershey Children's Hospital, Hershey, PA); Barry Markovitz, Jeff Terry, Rica Morzov (Children's Hospital Los Angeles, Los Angeles, CA); Vicki Montgomery, Andrew Michael, Melissa Thomas (University of Louisville and Kosair Children's Hospital, Louisville, KY); Marcy Singleton, Dean Jarvis, Sholeen Nett (Dartmouth Hitchcock Medical Center, Lebanon, NH); Douglas Willson, Michelle Hoot (Virginia Commonwealth University, Richmond, VA); Melania Bembea, Alvin Yiu (Johns Hopkins University, Baltimore, MD); David McKinley, Elizabeth Scarlett, Jennifer Sankey, Minal Parikh (Geisinger, Danville, PA); E. Vincent S. Faustino (Yale School of Medicine, New Haven, CT); Kelly Michelson, Jay Rilinger, Laura Campbell (Ann \& Robert H. Lurie Children's Hospital of Chicago, Chicago, IL); Shira Gertz (Hackensack University Medical Center, Hackensack, NJ); Jill M. Cholette (University of Rochester, Rochester, NY); Asumthia Jeyapalan (Holtz Children's Hospital-Jackson Memorial Hospital, Miami, FL); Margaret Parker (Stony Brook University, Stony Brook, NY); and Scot Bateman, Amanda Johnson (UMass Memorial Children's Medical Center, Worcester, MA).

\section{REFERENCES}

1. Whitaker BI, Rajbhandary S, Harris A: Trends in United States blood collection and transfusion: Results from the 2013 AABB Blood Collection, Utilization, and Patient Blood Management Survey. Transfusion 2016; 2173-2183

2. Puetz J, Witmer C, Huang YS, et al: Widespread use of fresh frozen plasma in US children's hospitals despite limited evidence demonstrating a beneficial effect. J Pediatr 2012; 160:210-215.e1

3. Karam O, Demaret P, Shefler A, et al; Canadian Critical Care Trials Group (CCCTG); Pediatric Acute Lung Injury and Sepsis Investigators (PALISI); BloodNet; PlasmaTV Investigators: Indications and effects of plasma transfusions in critically ill children. Am J Respir Crit Care Med 2015; 191:1395-1402
4. Stanworth SJ, Grant-Casey J, Lowe D, et al: The use of fresh-frozen plasma in England: High levels of inappropriate use in adults and children. Transfusion $2011 ; 51: 62-70$

5. Pandey S, Vyas GN: Adverse effects of plasma transfusion. Transfusion 2012; 52(Suppl 1):65S-79S

6. Holcomb JB, Tilley BC, Baraniuk S, et al; PROPPR Study Group: Transfusion of plasma, platelets, and red blood cells in a $1: 1: 1 \mathrm{vs}$ a $1: 1: 2$ ratio and mortality in patients with severe trauma: The PROPPR randomized clinical trial. JAMA 2015; 313:471-482

7. Spinella PC, Frazier E, Pidcoke HF, et al: All plasma products are not created equal: Characterizing differences between plasma products. J Trauma Acute Care Surg 2015; 78:S18-S25

8. Karam O, Lacroix J, Robitaille N, et al: Association between plasma transfusions and clinical outcome in critically ill children: A prospective observational study. Vox Sang 2013; 104:342-349

9. Inaba K, Branco BC, Rhee P, et al: Impact of plasma transfusion in trauma patients who do not require massive transfusion. J Am Coll Surg 2010; 210:957-965

10. Bochicchio GV, Napolitano L, Joshi M, et al: Outcome analysis of blood product transfusion in trauma patients: A prospective, riskadjusted study. World J Surg 2008; 32:2185-2189

11. Subramaniam K, Spilsbury K, Ayonrinde OT, et al: Red blood cell transfusion is associated with further bleeding and fresh-frozen plasma with mortality in nonvariceal upper gastrointestinal bleeding. Transfusion 2016; 56:816-826

12. Khan $\mathrm{H}$, Belsher J, Yilmaz M, et al: Fresh-frozen plasma and platelet transfusions are associated with development of acute lung injury in critically ill medical patients. Chest 2007; 131:1308-1314

13. Park PK, Cannon JW, Ye W, et al: Transfusion strategies and development of acute respiratory distress syndrome in combat casualty care. J Trauma Acute Care Surg 2013; 75:S238-S246

14. Müller MC, de Haan RJ, Vroom MB, et al: Evaluation of a multi-center randomised clinical trial on prophylactic transfusion of fresh frozen plasma: Implications for future trials. Transfus Med 2014; 24:292-296

15. Piccin A, Cronin M, Brady R, et al: Transfusion-associated circulatory overload in Ireland: A review of cases reported to the National Haemovigilance Office 2000 to 2010. Transfusion 2015; 55:1223-1230

16. Sarani B, Dunkman WJ, Dean L, et al: Transfusion of fresh frozen plasma in critically ill surgical patients is associated with an increased risk of infection. Crit Care Med 2008; 36:1114-1118

17. Johnson JL, Moore EE, Kashuk JL, et al: Effect of blood products transfusion on the development of postinjury multiple organ failure. Arch Surg 2010; 145:973-977

18. Haubelt $\mathrm{H}$, Blome $M$, Kiessling $\mathrm{AH}$, et al: Effects of solvent/detergenttreated plasma and fresh-frozen plasma on haemostasis and fibrinolysis in complex coagulopathy following open-heart surgery. Vox Sang $2002 ; 82: 9-14$

19. Krusius T, Auvinen M-K, Nikkinen L: Introduction of octaplas - in clinical use decreased the rate of severe adverse reactions. Vox Sang 2010; 99(Suppl 1):461

20. Flesland O: A comparison of complication rates based on published haemovigilance data. Intensive Care Med 2007; 33(Suppl 1):S17-S21

21. Bindi ML, Miccoli M, Marietta M, et al: Solvent detergent vs. fresh frozen plasma in cirrhotic patients undergoing liver transplant surgery: A prospective randomized control study. Vox Sang 2013; 105:137-143

22. Whitaker BI, Hinkins S: Report of the US Department of Health and Human Services. The 2011 national blood collection and utilization survey report. Washington, DC: U.S. Department of Health and Human Services, Offices of the Assistant Secretary for Health, 2011 